\title{
Virtual Reality Exposure Simulation for Student Veteran Social Anxiety and PTSD: A Case Study
}

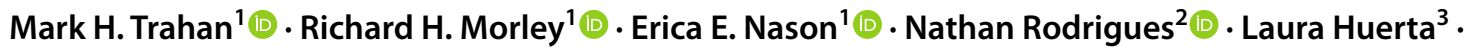 \\ Vangelis Metsis ${ }^{3}$
}

Accepted: 6 December 2020 / Published online: 19 January 2021

(c) The Author(s), under exclusive licence to Springer Science+Business Media, LLC part of Springer Nature 2021

\begin{abstract}
Exposure based exercises are a common element of many gold standard treatments for anxiety disorders and post-traumatic stress disorder and virtual reality simulations have been evaluated as a platform for providing clients with opportunities for repeated exposure during treatment. Although research on virtual reality exposure therapy (VRET) indicates effectiveness and high levels of user satisfaction, VRETs require a participant to complete exposure exercises in-offices with specialized equipment. The current exploratory case method study evaluates the experience and outcomes of one student veteran with social anxiety disorder and PTSD completing twelve sessions of VRET exposure using a mobile phone simulation of a virtual grocery store. The participant reported decreases in psychological symptoms, improvements in neurological connectivity, and better sleep quality upon completing the trial. Results suggest that VRET using a mobile application is feasible and warrants further research to evaluate effectiveness more fully. Implications include the use of a mobile based virtual reality simulation for intervening in social anxiety for student veterans.
\end{abstract}

Keywords Virtual reality $\cdot$ Exposure therapy $\cdot$ Simulation $\cdot$ Social anxiety $\cdot$ PTSD

\section{Introduction}

Virtual Reality Exposure Therapy (VRET) is an alternative form of behavioral therapy that has demonstrated initial clinical efficacy in reducing PTSD symptoms and can significantly impact assessing and treating psychological disorders (Gonçalves et al. 2012). Exposure therapy focuses on modifying response behaviors such as avoidance to situations or thoughts and memories that may be anxiety-provoking or challenging through repeated exposure to feared stimuli. Considered as a first-line treatment for PTSD and anxiety disorders, exposure therapy has shown to be highly effective according to numerous guidelines (e.g., McLean

Mark H. Trahan

marktrahan@txstate.edu

1 School of Social Work, Texas State University, 601 University Blvd., Encino Hall \#158, San Marcos, TX 78666, USA

2 Department of Respiratory Care and Texas Sleep Center, Round Rock, TX 78665, USA

3 Department of Computer Science, Texas State University, San Marcos, TX 78666, USA and Foa 2011; Schmidt and Keough 2010). Emotional Processing Theory may explain the mechanisms underlying exposure therapy (Foa and Kozak 1986). Triggered upon confrontation with trauma-related information, the phobic fear structure is activated. Thus, mechanisms for symptom reduction involve recurring confrontation with anxietyprovoking stimuli (in vivo or imaginal) that elicit the fear structure's activation to attain habituation and eliminate fearful and anxious reactions (Kothgassner et al. 2019). As a result, VRET aids in modifying dysfunctional assessments of threat by reducing the conditioned response associated with the anxiety-inducing stimuli over time during exposure (Boeldt et al. 2019).

VRET uses the key principles from traditional exposure therapy and incorporates real-time 3-D graphics and visual displays to immerse participants in a computer-generated virtual environment, offering an adaptable treatment for psychological disorders that promotes new learning and assistance in training, assessing, and delivering psychotherapy skills (Boeldt et al. 2019). Generally, the virtual environment is intended to help patients confront fearful environments and situations that may not be safe to encounter or are difficult to access in real life scenarios. Offering a unique ability 
to stimulate environments, VRET can challenge the boundaries of everyday surroundings (Kothgassner et al. 2019). VRET is especially useful as it permits repeated exposure infinitely with an adaptable and individualized immersive environment easily implemented by therapists and often more accessible and acceptable to patients than in vivo exposure (Boeldt et al. 2019). VRET further carries the advantage of increased control over stimuli as therapists can see what patients see in real-time, which addresses limitations of imaginal exposure, such as the patients' inability to form mental images. This advantage allows therapists to guide patients through the VRET while monitoring and supporting the patient, thus presenting VR as a valuable tool for mental health therapy by providing patients with a physiologically and emotionally evocative experience (Jerdan et al. 2018). Patients report engaging experiences that feel realistic during VRET (Freeman et al. 2017).

With recent changes in provision of psychological services related to COVID-19 including social distancing and the use of telehealth for providing interventions in clinical social work, a mobile based virtual reality phone application is timely and important. The cost of mental health mobile applications is generally more accessible than in person mental health services (Ebert et al. 2018). Because of the ability for increased access potential, mobile phone applications have often become a resource for mental health interventions from anxiety to depression to PTSD (Linardon et al. 2019). Mental health mobile phone applications are cost effective and may reach clients who are reticent to seek out in person services (Jones et al. 2015). For populations that may not be able to access a desktop computer or the internet due to financial constraints, mobile phones provide greater potential for access (Dolan 2016). Costs associated with this intervention include a low-cost VR headset (\$10-30) and the cost of the mobile application. Because of cost and accessibility, mobile based VR applications provide great promise to clinical social workers for mental health intervention.

VRET research has proliferated exploring its use with psychological disorders, especially anxiety disorders (Carl et al. 2019). Early VRET studies on veterans with chronic and treatment-resistant PTSD have yielded promising results for VR-based exposure (Rothbaum et al. 1999, 2001). More recently, a qualitative review concluded that VRET is a potentially beneficial tool for treating different types of trauma (Gonçalves et al. 2012). Although over two decades of research documents the effectiveness of VRET for anxiety disorders, exposure therapy remains an underutilized treatment for anxiety disorders, at a time when effective and accessible treatment is critical (Boeldt et al. 2019). When presented as a scalable tool, VRET can increase access to effective exposure therapy, improving the treatment of psychological disorders, especially anxiety disorders. Incorporating VR in the therapeutic process provides patients with an evidence-based anxiety treatment that can improve access, acceptability, and effectiveness of therapy. In the current study, participants were immersed in a virtual realitybased noncombat environment, a grocery store, that stimulated anxiety and PTSD symptoms in post-combat veterans to assess the effectiveness of VRET in increasing tolerance to anxiety based cues and decreasing symptoms. This intervention was delivered through a mobile phone application platform, providing participants with the flexibility to utilize the intervention at convenience.

\section{Virtual Reality Exposure Therapy for Student Veterans}

Avoidance of stimuli associated with traumatic events, a diagnostic criterion of PTSD, and social anxiety, or an intense fear of being watched or judged by others, are often comorbid and may increase the risk of suicidality along with decrease quality of life and mental health (McMillan et al. 2017). For returning combat veterans, assessment of risk associated with being in crowds, along with difficulty with interactions with others due to fear of being watched or judged, may result in avoidance of places that are particularly stimulating for anxiety. The combination of these comorbid conditions appears to be additive, resulting in reduced potential for social support (McMillan et al. 2017), an important contributor to recovery from PTSD (Wall and Lowe 2020). For student veterans, social anxiety and avoidance can produce significant distress, particularly when acclimating to large crowded campuses requiring daily frequent social interaction to navigate student responsibilities of attending classes and studying with peers. Student veterans report that social anxiety may significantly increase in crowded walkways, chaotic public areas, bus transportation, and cafeterias or campus restaurants (Trahan et al. 2019). Additionally, student veterans report challenges with daily life functioning in other venues where people congregate, including grocery stores, restaurants, concerts, sporting events, and university events such as graduations (Trahan et al. 2019). The avoidance of these environments by veterans may reduce their campus presence, increase anxiety resulting in reduced potential for learning, and contribute to increased attrition rates prior to graduation.

As social avoidance of crowded places generally contributes to lack of utilization of campus activities, our previous work focused on producing a virtual reality environment that would be usable and acceptable for student veterans to acclimate to social situations through exposure and tolerance. With veteran avoidance of crowded spaces, we recognized that virtual spaces with crowds might allow veterans the opportunity to acclimate and navigate social environments. From qualitative interviews with veterans (Trahan et al. 
2019), our team identified scenarios and specific cues to anxiety within these crowded spaces. These cues for anxiety were then communicated to a team of graphic design and computer science students, who developed the virtual space with multiple iterations of student and social work faculty feedback sessions designed to review development progress and hone design to implement these cues. Several scenarios, including a crowded university walkway, an elevator, and a grocery store were then prototyped with veterans (Metsis et al. 2019). Using this rapid prototyping process, the final grocery store design was identified as the most generalizable and feasible environment for working with veterans to address social anxiety. Furthermore, it was also identified as a location that would produce moderate to high levels of social anxiety (Nason et al. 2019). The final grocery store's design included various triggers for social anxiety, including sounds of a crying baby, people crowding aisles, disagreements between customers, a dropped backpack on the floor, and a checkout counter with cashier interacting with the customer (Fig. 1). Once the grocery store was developed, the program was transferred to a mobile-based application, that could be uploaded to a user mobile phone, providing ease of at-home access, with limited equipment necessary to use the intervention.

\section{Neuroimaging}

Although research has demonstrated the VRET is an effective treatment option, little research has examined the neurological mechanisms that are implicated in reducing

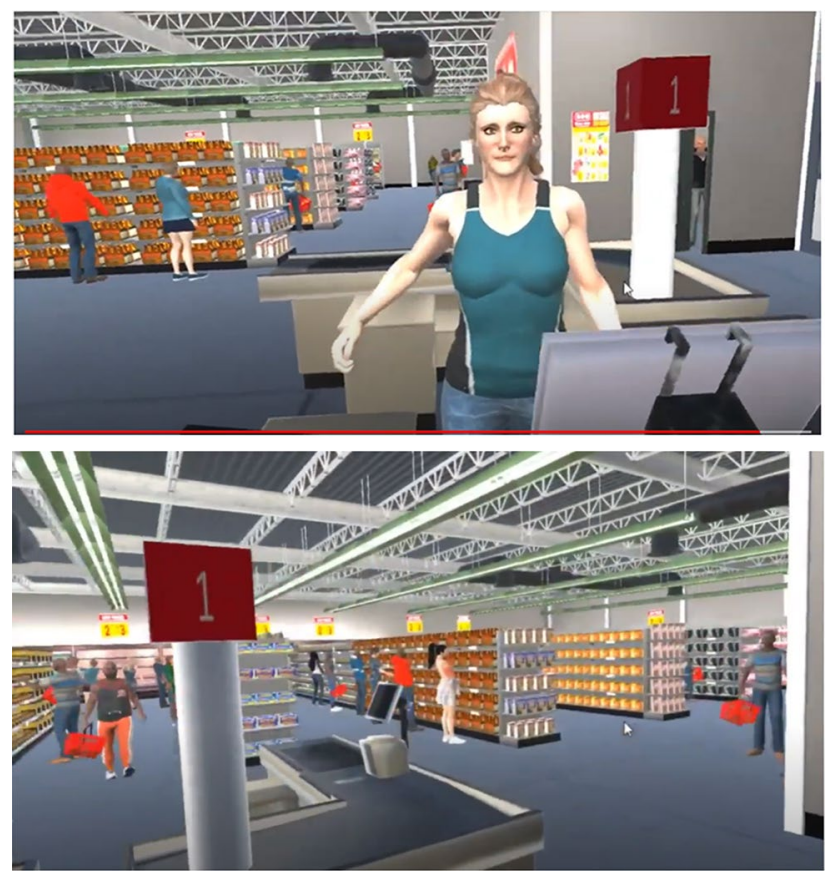

Fig. 1 Virtual reality grocery store with cues for social anxiety symptoms with the intervention. Research findings suggest that three intersecting large-scale brain networks, including the Salience Network (SN), the Default Mode Network (DMN), and the Central Executive Network (CEN), mediate the brain's link to behavior, including symptomatic behavior associated with several mental health diagnoses (Bressler and Menon 2010). While researchers have not explicitly studied the intersection of brain networks with subjects diagnosed PTSD and social anxiety, research findings do indicate that both social anxiety and PTSD are characterized by disrupted interconnectivity between the DMN and CEN stemming from an overactive SN (Sripada et al. 2012; $\mathrm{Xu}$ et al. 2019). Indeed, the $\mathrm{SN}$ is involved in many tasks associated with PTSD and social anxiety, including attention, threat detection, arousal, and emotional regulation (Barrett and Satpute 2013; Morley et al. 2019; Seeley et al. 2007). Research indicates the SN is inversely correlated and modulates the CEN and the DMN (Barrett and Satpute 2013; Chiong et al. 2013; Seeley et al. 2007). This dysregulation leads to an impaired extinction of fear (Chen et al. 2018; Garret et al. 2019; Wolf and Herringa 2016). Within these large-scale networks are key nodes that anchor the large scale networks including the medial prefrontal cortex (mPFC), posterior cingulate cortex (PCC), and precuneus within the DMN, the anterior cingulate cortex (ACC) and anterior insular cortex (AIC) within the $\mathrm{SN}$, and dorsolateral prefrontal cortex (DLPFC), Posterior Parietal Cortex (PPC) within the CEN (Menon 2011; van den Heuvel and Sporns 2011, 2013).

Research findings indeed indicate that disrupted interbrain network connectivity stems from overactivation in the amygdala, a region vital to fear conditioning (Chen et al. 2018; Garret et al. 2019; Sripada et al. 2012). More specifically, research findings indicated that stress-induced hormone cortisol modulates activation in the amygdala and is associated with increased connectivity within the SN and decreased connectivity in the DMN (Garret et al. 2019; van Stegeren et al. 2007). Research also shows that trauma leads to increased amygdala sensitivity (Chen et al. 2018). Trauma also leads to increased amygdala connectivity to the precuneus, which is a region vital for episodic memory and a key node of the DMN, and decreased connectivity between the precuneus and the medial prefrontal cortex (mPFC), which is a key region for modulating the amygdala and resilience to trauma (Chen et al. 2018; Rauch et al. 2005).

Indeed, findings indicate that an impaired $\mathrm{mPFC}$ is a key neurological feature associated with neurological dysfunction with regulating trauma and fear (Rauch et al. 2005). Evidence suggests that PTSD symptoms were associated with reduced connectivity between the $\mathrm{mPFC}$ and the DLPFC, a key node of the CEN (Chen et al. 2018; Wolf and Herringa 2016). Research also suggests that negative affect among people with PTSD is associated with increased 
amygdala activity, decreased mPFC activity, and that suppression of negative emotions is associated with the anticorrelation between the mPFC and amygdala (Delgado et al. 2008; Etkin and Wager 2007; Johnstone et al. 2007; Urry et al. 2006). Moreover, research findings indicate that this disrupted anticorrelation was associated with worsening symptoms over time (Wolf and Herringa 2016).

While the mPFC may be important for fear extinction characteristic of both social phobia and PTSD, the increased amygdala and salience network association with the precuneus and PCC play a specific role in social phobia. Indeed, these areas of the DMN play a key role in both episodic memory and theory of mind (Cavanna and Trimble 2006). Given the amygdala and the SN are associated with fear and threat detection with processing theory of mind, these findings seem to indicate the increased tendency of associating understanding others with fear, which is characteristic of social phobia. Given that traumatic events lead to this increased association between regions associated and associated with fear, understanding others, and decreased resilience to fear, evidence indicates a common neurodevelopmental pathway for social anxiety and PTSD. This common developmental pathway would suggest why these two diagnoses are often comorbid.

Given that these two diagnoses have a common neurodevelopmental pathway, pursuing an intervention that targets disrupted brain network connections could be useful in treating both conditions. Previous research indicates that exposure therapy might be an effective tool to enhance connections disrupted by exposure to violence (King et al. 2016, 2017). Indeed, King et al. (2016) found that exposure therapy is linked to increased connectivity between the posterior cingulate cortex and the dorsal lateral prefrontal cortex. King et al. (2016) also found that this improved connectivity was correlated with a decrease in avoidance and hyperarousal symptoms. King et al. (2017) found that exposure therapy is linked to increased activity in the mPFC and DLPFC during an emotional face task, correlated with reduced symptoms of PTSD. These findings together indicate that exposure therapy reduces the association of the salience network and precuneus in response to a traumatic event, improved connections within the DMN, and enhanced activation of the $\mathrm{mPFC}$ in response to regulating fear.

\section{Sleep Quality}

Sleep and mental health are interconnected. Sleep complaints are ostensibly ubiquitous within PTSD and anxiety disorders and are often associated with hyperarousal (Richards et al. 2019). Nighttime sleep disturbances are the most salient conveyed sleep complaints in PTSD, having been reported in as many as $90 \%$ of cases (Harvey et al. 2003). Sleep disturbances lead to fractured sleep, poor sleep quality, daytime complaints, and the subsequent exacerbation of deleterious concomitant symptoms (Harvey et al. 2003; Mclay et al. 2010). VRET is an effective treatment for reducing symptoms of PTSD and anxiety disorders (Gonçalves et al. 2012), and postulates an auspicious mechanism vis-a-vis improving parallel sleep disturbances and concomitant symptoms.

\section{Methods}

For this case study, we implored utilized an exploratory instrumental case study methodology (Crowe et al. 2011; Yin 1984), as the purpose of the study was to better understand how simulation-based exposure to cue related stress triggers social anxiety for student veterans. While in vivo is a gold standard for exposure therapy, virtual reality provides a highly accurate simulation-based learning environment that may effectively stimulate an anxiety response, which may be attenuated with repeated exposures or a treatment (Chesham et al. 2018). The phenomenon of the stimulation of anxiety within a pre-designed virtual environment for addressing a specific psychopathology has merit for future therapeutic interventions. In determining the case to be selected, the research team engaged in an iterative process of discussion and consensus. The primary author made a recommendation about the case and received feedback from each researcher. The basis of selection was consistency of case as compared to other cases, including a moderate to high social anxiety score, the presence of PTSD which is often co-morbid with social anxiety disorder, the selection of an older student veteran (more common to the university system), dose validation, and no cybersickness symptoms. To collect data, the researchers utilized both quantitative (questionnaires, EEG) and qualitative (interviews) research methods, although the qualitative summary is not reported here. The research question was "How can a mobile phone delivered virtual reality simulation of a grocery store be utilized to provide a realistic environment for repeated exposures to induce social anxiety symptoms and reduce response with habituation?"

\section{Case Example Study}

This evaluation of a mobile virtual reality intervention was approved by the university Institutional Review Board. "Rick", a 36-year-old white male student veteran, initiated participation in the project by responding to a recruitment email delivered to the student veteran population at the university. The participant signed an electronic informed consent at the time of initiation through an online prescreen. Rick completed the online prescreen through Qualtrics in 12:28 min in April 2019. The prescreen included 
demographic questions including military history, medical history, social anxiety and distress scale, the PTSD Checklist for the DSM-V [PCL-5; Weathers et al. 2013], and the Symptom Checklist-90-Revised [SCL-90; Prinz et al. 2013]. The student reported that he was currently married, residing with his wife and two children. He stated that he was previously an active duty Marine, with four years of service and one previous deployment, eight months in length. He reported that he was unemployed, identifying as a full-time student in his senior year of undergraduate education. He denied a history of a traumatic brain injury, seizures, mobility issues, and denied taking medications. The student did report a history of substance use disorder and stated that he had abstained from alcohol for two years prior. The student also reported in the prescreen that he had a history of PTSD, resulting in anxiety and panic attacks. Rick scored a 19 on the social anxiety and distress scale, indicating a high moderate level of social anxiety for a male (low $=<4$, intermediate $=4-19$, high $=20+$; Watson and Friend 1969). He also scored a 46 out of 80 on the PCL-5, above the generally accepted cutoff of 31-33, indicating the presence of PTSD (Blevins et al. 2015; Weathers et al. 2013).

Furthermore, a provisional diagnosis of PTSD was made based on scores in individual symptom clusters. The SCL90-R was scored to determine if psychotic symptoms were present, and the results were not indicative of psychotic symptoms. Upon reviewing the student's scores, demographics, and student status, it was determined that the student would be contacted to participate in the study.

After review and confirmation of inclusion, Rick was contacted via email about study involvement. Rick agreed to participate and was provided with an appointment time for an initial screening session. At first intake, he completed another set of screening measures, including the Pittsburgh Sleep Quality Index [PSQI; Buysse et al. 1989], Motion Sickness Questionnaire [MSQ; Frank et al. 1983], a PCL-5, a Tellegen Absorption Scale (Tellegen and Atkinson 1974), and a Five Face Mindfulness Scale (Baer et al. 2006). The PSQI was administered for the baseline collection of sleep data. As motion sickness history has been found to be associated with cybersickness (Gavgani et al. 2018), the MSQ was administered to determine the risk related to nausea, dizziness, and other motion sickness symptoms that may affect the use of the application, along with participant safety. The Tellegen Absorption Scale (Tellegen and Atkinson 1974) and the Five Facet Mindfulness Scale (Baer et al. 2006) serve to control for these variables in a larger study related to usability and feasibility of the intervention. Consistent with the screening questionnaire, the student's total score on the PCL-5 at this timepoint was 44. After completing these measures, the client was interviewed by a doctoral-level faculty psychologist using the mood, psychotic symptom, anxiety, trauma and stress related disorders, and sleep-wake disorder modules of the Structured Clinical Interview (Research Version) for the DSM-5 (SCID-RV, First et al. 2015). On the SCID, the individual met diagnostic criteria for current panic disorder with agoraphobia, social anxiety disorder, posttraumatic stress disorder, and insomnia disorder. The participant endorsed an extensive trauma history, both during military service and as a civilian. Rick reported current passive suicidal ideation but denied any current suicidal plans or intentions. Based on the information gathered, it appeared that his suicide risk was low and did not require additional intervention. Upon review of the SCID diagnostic criteria and the measures, the student met inclusion criteria, as he had social anxiety, no indication of psychotic symptoms, and no evidence of a history of TBI.

After meeting criteria for the study, Rick was evaluated for a baseline measure of biophysiological responses, including heart rate, galvanic skin response, and the client was fitted with a Brain Vision Live Amp 64-Channel Portable Electroencephalogram (EEG) system. Biometric information was collected utilizing BioRadio Wireless Physiology Monitor and associated peripherals from Great Lakes Neurotechnology. Due to the nature of social anxiety, researchers considered how to obtain a resting-state biophysiological baseline to evaluate the change in bio physiology upon exposure. It was determined that a brief mindfulness exercise upon arrival could assist participants in overcoming the anxiety of attending a session with multiple researchers that they had never met. Prior to the baseline resting-state evaluation, the participant was guided through a 3-min mindfulness exercise and then resting-state baseline data was collected using a portable EEG. After $10 \mathrm{~min}$ of baseline data on connectivity, the researchers then removed the EEG cap and proceeded to brief the participant on the VR application. Rick was provided with an overview of the intervention, and a tutorial about moving within the VR space in the app. The application was provided to the participant using a standard plastic VR headset with a 3-D VR grocery store loaded on a mobile phone. The plastic VR headset is designed to slide a mobile phone into the headset to view the VR environment. The mobile phone was thus placed in the VR headset, allowing the participant to view the virtual grocery store and complete a tutorial on how to move within the virtual space. As the intervention was delivered through a mobile phone application, there was not an option to provide a joystick for user movement. To adjust for this, the mobile application was designed so that tilting the headset slightly up or down by looking up or down would move the participant forward and backward. The participant was briefed about how to move within the grocery store and provided two minutes to practice. Rick was then provided with the following directions: "What you see here is a virtual grocery store. We would like to ask you to explore the grocery store for the next $10 \mathrm{~min}$. Every 30 seconds, we will ask you to 
rate your anxiety level from 0 to 100 . After 10 minutes, the simulation will end." The Subjective Units of Distress Scale (SUDS) ranging from 0 to 100 , was used to evaluate the intensity of anxiety symptoms throughout the exposure. This approach is consistent with other exposure-based therapies. During the exposure to the virtual grocery store, the participant was asked during intervals about self-reported stress using a 0-100 SUDS scale, which was documented. Additionally, biometric readings of heart rate and GSR were also recorded. After 10 min of exposure, the simulation ended.

After exposure, Rick completed a virtual reality presence questionnaire (Witmer \& Singer 1998) to determine his level of engagement in the app. The presence questionnaire provides a baseline score related to level of engagement with the app. The participant was briefed about the intervention and instructed to utilize the VR app at least three times per week for four weeks, for 12-15 min per session. This dose is consistent with previous research related to effective VR interventions for social anxiety (Chesham et al. 2018), and consistent with previous research that increased exposure doses result in greater decreases in symptoms (Powers and Emmelkamp 2008; Opris et al. 2012). An appointment was then set for Rick to return in two weeks to check on dosage and use, recharge the sensor, collect usability data, and to address any questions or concerns that had arisen in the process of utilizing the intervention and navigating the virtual space.

Rick returned to meet with the research team after two weeks utilizing the intervention. He reported that he had utilized the intervention three times per week, for 12-15 min per session for both weeks. He reported no difficulties with accessing or utilizing the intervention. He completed a System Usability Scale (Brooke 1996), of which he scored the mobile technology as a 95 out of 100, indicating a system that is highly usable. Two additional questions were asked about usability regarding difficulty with opening the application and the ability to move within the virtual space, both of which he reported were, "very easy." An appointment for a follow-up session was scheduled for two weeks after this date. After the session, Rick was sent a gift card of $\$ 100$ as renumeration for utilizing the intervention for the first two weeks.

Rick returned for a final data collection session after four weeks of utilizing the intervention. He stated that he had used the intervention at the prescribed dosage, 12-15 min, three times per week for two additional weeks, as previously accomplished in the first two. He completed several scales for the purpose of measuring changes in social anxiety, PTSD symptoms, and sleep. After the completion of the measures, the student was fitted with an EEG cap. After putting on this cap, the research assistant fitted him with technology to assess biomarker data. The participant was provided with a 3-min mindfulness relaxation meditation, equivalent to the meditation provided in the first session. After completing this activity, $10 \mathrm{~min}$ of baseline restingstate data was collected from the research participant. During this time, the participant was instructed to attempt to stay as motionless as possible to collect the data. After the completion of this data collection period, the EEG cap was removed from the participant. The participant was then provided with the VR unit, including the mobile phone and the plastic VR glasses, to utilize the mobile phone VR grocery store app and collect information about self-reported stress levels. Equivalent to the first session, the participant entered the VR space, and was prompted every 30 seconds to provide a SUDS score of his level of distress. This information was recorded by one of the researchers. Upon completion of the 10-min exposure exercise, the participant was asked to remove the VR goggles and the physiological monitoring technology was also removed. At this time, the participant answered questions related to the system usability along with two questions pertaining to usability that were added.

Review of the SUDS data indicated a reduction in selfreported stress during exposure to the application. The original mean of self-reported stress $(M=33)$ was greater than the mean of self-reported stress after the intervention $(M=14.5)$, although a t-test failed to indicate a significant difference between the two time points $[t(45)=3.00$, $p=0.205]$. However, on measures of social anxiety, PTSD, and sleep quality, there were significant differences in these scores from baseline to post-intervention. The participant scored a nine on the Social Anxiety and Distress scale, indicating a $52.6 \%$ reduction in social anxiety in over four weeks. The participant also scored a 37 out of 80 on the PCL-5, an $11 \%$ reduction of PTSD symptoms. These reductions indicate a drop in both social anxiety and PTSD.

EEG data were preprocessed and analyzed using the brain analyzer 2.0 analysis software (Mideksa 2018). All data were preprocessed to remove artifacts using a low filter pass of $0.53 \mathrm{~Hz}$ and a high pass of $60 \mathrm{~Hz}$ and ocular correction ICA to remove artifacts associated with blinking. The analysis included hemisphere specific posterior cingulate to mPFC and current source density analysis of the bivariate mPFC. Regions of interest were located using the Analyzer 2 Loreta Program using the following MNI coordinates right mPFC $652-2$, left mPFC $-652-2$, right PCC $8-56$ 26, and left PCC $-8-5626$ (Andrews-Hanna et al. 2010). The results showed that post-test connectivity coefficients were significantly higher in the left $(t(1)=12.92, p=0.017)$ and higher but almost statistically significance in the right $(t(1)=3.95, p=0.078)$ hemisphere. Moreover, the source density analysis showed larger amplitudes MPFC electrical activity in the left $\left(7.38 \times 10^{-4}-3.04 \times 10^{-4}\right)$ and right $\left(6.87 \times 10^{-4}-2.74 \times 10^{-4}\right)$ hemispheres.

For sleep data, pre-and-post intervention findings are presented in Table 1 . The participant's sleep improved with a 
Table 1 Pre-and-post intervention comparison of global and component PSQI scores

\begin{tabular}{lllc}
\hline Component & $\begin{array}{l}\text { Pre-inter- } \\
\text { vention }\end{array}$ & $\begin{array}{l}\text { Post-inter- } \\
\text { vention }\end{array}$ & Difference \\
\hline Overall global PSQI & 10 & 6 & -4 \\
Subjective sleep quality & 2 & 1 & -1 \\
Sleep latency & 1 & 1 & 0 \\
Sleep duration & 2 & 1 & -1 \\
Habitual sleep efficiency & 0 & 0 & 0 \\
Sleep disturbances & 2 & 2 & 0 \\
Sleep medication use & 0 & 0 & 0 \\
Daytime dysfunction due to & 3 & 1 & -2 \\
$\quad$ sleepiness & & \\
Lower scores are indicative of healthier sleep & \\
\hline
\end{tabular}

Table 2 Electrophysiological connectivity coefficient correlation tables

\begin{tabular}{|c|c|c|c|c|}
\hline & Left MPFC & Right MPFC & Left PCC & Right PCC \\
\hline \multicolumn{5}{|l|}{ Pre-test } \\
\hline Left MPFC & 1 & & & \\
\hline Right MPFC & $.98 * *$ & 1 & & \\
\hline Left PCC Pre & $.10 * *$ & $.094 * *$ & 1 & \\
\hline Right PCC P & $.106^{* *}$ & $.097 * *$ & $.95 * *$ & 1 \\
\hline \multicolumn{5}{|l|}{ Post-test } \\
\hline Left MPFC & 1 & & & \\
\hline Right MPFC & $.99 * *$ & 1 & & \\
\hline Left PCC Pre & $.90 * *$ & $.90 * *$ & 1 & \\
\hline Right PCC P & $.91 * *$ & $.91 * *$ & $.85^{* *}$ & 1 \\
\hline
\end{tabular}

*.05; **.01

Table 3 Source density MPFC activity amplitudes

\begin{tabular}{ll}
\hline Right hemisphere & MPFC \\
\hline Pre-test & $2.74 \times 10^{-4}$ \\
Post-test & $3.04 \times 10^{-4}$ \\
Left hemisphere & MPFC \\
Pre-test & $6.87 \times 10^{-4}$ \\
Post-test & $7.38 \times 10^{-4}$ \\
\hline
\end{tabular}

four-point change, reflecting improvements in three of the seven sleep component scores. While the post intervention score does not meet a global index indicating "good sleep", it does indicate positive progress towards overall sleep quality. The overall global PSQI score (overall sleep quality) improved by four points post-intervention, reflecting improvements in three of the seven sleep component scores (Tables 2, 3). Subjective sleep quality improved one point from a score of "2" (moderate difficulty) to a score of " 1 " (mild difficulty), sleep duration improved one point from a score of " 2 " (moderate difficulty) to a score of "1"

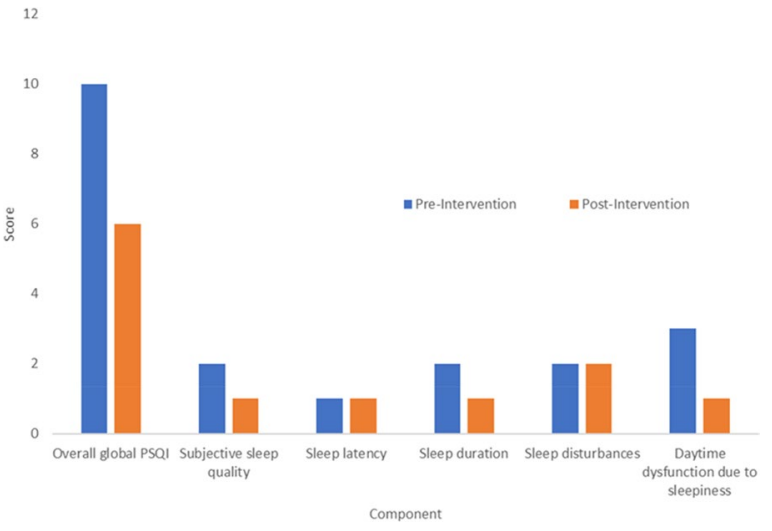

Fig. 2 Visual representation of pre and post intervention global and component PSQI scores. Lower scores are indicative of healthier sleep. Component scores of zero (no difficulty) pre-and-post intervention have been removed from the figure

(mild difficulty), and daytime dysfunction due to sleepiness improved two points from a score of "3" (severe difficulty) to a score of "1" (mild difficulty). Pre-and-post intervention scores were maintained for the remaining sleep components: sleep disturbances remained at a score of "2" (moderate difficulty), and sleep latency remained at a score of " 1 " (mild difficulty). No sleep component score exhibited deterioration. A visual representation of pre-and-post intervention findings are presented in Fig. 2. It is also imperative to note that the participant did not report meeting the American Society for Sleep Medicine (AASM) recommended amount of seven hours of sleep duration (Watson et al. 2015) per night at any time during the study period.

\section{Discussion}

Rick presented with comorbid social anxiety disorder, panic disorder with agoraphobia, posttraumatic stress disorder, and insomnia disorder. His clinical presentation was complex in nature and he had been experiencing anxiety symptoms for over a decade. His clinical presentation is likely to be consistent with the types of comorbidities encountered in outpatient settings. For this specific case example, mobilebased virtual reality exposure therapy to address veteran social avoidance and anxiety resulted in statistically significant decreases in social anxiety and PTSD and an increase sleep quality between the pre- and post- follow up assessments. He also noted decreases in his stress levels during exposure, though the change was not statistically significant. Without therapeutic supervision during exposures, the case illustrates how simulation-based exposure through an empirically designed intervention developed specifically to induce social anxiety symptoms can provide a platform for 
reducing social anxiety through repeated exposures. As this intervention was developed specifically for the purpose of inducing these symptoms, including inducing auditory and visual cues, this case example demonstrates how mobile phone technology and the use of virtual simulations can be developed to provide a platform for conditioning related to anxiety based cues.

This case study illustrates neuroscience research findings suggesting a common neurological pathway underlining social anxiety and PTSD. Previous results that have linked both social anxiety and PTSD are associated with increased precuneus aligning with the amygdala and salience network, decreased connectivity between key nodes of the DMN, and weakening the mPFC function. The participant showed increased connectivity between key nodes of the DMN and increased amplitudes electrical activity in the MPFC during the post-test. These changes in neurological activity are linked to a reduction in symptoms and improved sleep. Given that reduced DMN connectivity is also associated with sleep deprivation (De Havas et al. 2012), these findings point to the potential impact of enhanced DMN function as a treatment efficacy for treating symptoms of PTSD and improving resiliency in fear-based disorders. This case study provides an in depth illustration of the use of mobile VR applications for the delivery of exposure-based treatment of anxiety disorders. More specifically, this case illustrates that improved connectivity between key nodes in the DMN are linked to improved rest and increased activity in the MPFC. Given that the MPFC is a key region for regulating fear, this study demonstrates how increased electrical amplitudes in MPFC may result in a reduction of symptoms of fear.

Despite the participant not meeting scoring criteria at or below the poor sleep quality threshold post-intervention, the participant did improve their overall global PSQI (sleep quality) score by four points, representing very optimistic and encouraging findings for future research endeavors incorporating the aforementioned protocols. Poor sleep quality has previously been found to correspond with reduced amygdala-posterior cerebellar lobe connectivity in individuals with anxiety and depression (Klumpp et al. 2018). Other studies have shown that sleep duration deprivation has resulted in a sixty-percent greater magnitude or amygdala reactivity and a significant loss of functional connectivity between the amygdala and the medial prefrontal cortex (Yoo et al. 2007). Additionally, student veteran populations may have higher manifestation rates of insomnia than other populations (Rodrigues et al. 2019), and studies of sleep deprivation and insomnia disorder suggest sleep loss may modify amygdala-frontal connectivity. Taken together, findings suggested shared neurobiology between sleep and emotional systems; however, the impact of sleep quality on the amygdala circuit in anxiety or depression is unclear (Klumpp et al. 2018), and further research is recommended.

\section{Limitations and Future Research}

As with all case studies, there are several limitations related to the generalizability of the presented results. First, case study is a relevant research design when attempting to understand questions of "how" and "why" (Yin 1984) and is not generalizable. As this project utilized quantitative pre and post data to evaluate one case, we suggest that this individual case study may not thoroughly answer the "how" question pertaining to this intervention. Further research, perhaps using qualitative methods, is necessary to understand specific factors within the exposure to the scenario that trigger social anxiety, providing a more complex and robust understanding of the question of how virtual reality may provide a more realistic intervention platform for addressing anxiety. While we also attempted to connect the neuroimaging with associated stress and sleep outcomes, we cannot state that these outcomes are caused through changes in neuro connectivity. Furthermore, we also recognize that individual preferences within the virtual space may promote a variety of responses. For instance, participants may prefer different areas of the store, leading to differences in changes of pre-post data.

In recent years, questions about the dosage and timing of clinical interventions have become increasingly common in clinical trials. For example, recent research has compared the effectiveness of exposure-based protocols for posttraumatic stress disorder, such as Prolonged Exposure, when using a massed (e.g., the client is seen daily and completes the treatment protocol over a period of two weeks) versus spaced (e.g., the client is seen using a more traditional session spacing of once or twice per week over the course of several months) (Foa et al. 2018). A limitation to our study was a lack of real time tracking of how much time was spent in the virtual application, along with areas visited within the scenario. Participants reported to the research staff at each time point the amount of time and days spent in the application. Thus, participants seeking social desirability may not have been fully forthright about the time spent within the application. This research has found that massed treatment may be more effective in the short-term and noninferior in the long-term compared to more traditionally timed exposure exercises. Mobile applications allowing individuals to engage in exposure exercises at home allow for flexibility that could increase engagement in exposure and reduce symptoms over shorter periods of time compared to currently available, in-office virtual reality exposure exercises for anxiety disorders (Kim et al. 2017). However, future research is needed to systematically evaluate the ideal timing and dosage of mobile VRET in a larger sample. 


\section{Clinical Implications}

Many of the gold standard treatments for anxiety and posttraumatic stress disorders require individuals to engage in vivo exposure exercises outside of therapy sessions. These exercises are intended to help individuals habituate to feared stimuli in real-world settings, thus, facilitating a habituation response. Past research has demonstrated that VRET is likely as effective as in vivo exposure (Wechsler et al. 2019). One limitation of traditional VRET is that it requires that individuals have access to specialized equipment and virtual exposure might be limited to in-office sessions. Mobile-based VRET allows users to engage in regular exposure exercises from a safe environment (e.g., at home). This both allows for more frequent trials and increased control that could contribute to higher levels of acceptability and feasibility than in vivo exposure or in-office VRET. Furthermore, mobile based VRET provides participants with opportunities for exposure based on convenience, allowing participants flexibility to receive repeated exposure when feasible for the user.

Additionally, this mobile-based technology appears to have both psychological and neurophysiological benefits, resulting in a reduction of social anxiety for this veteran. Clinical interventions utilizing virtual reality exposure therapy for reducing social avoidance require discernment from a clinician. With this veteran, a mobile-based virtual reality grocery store provided stimuli such as crowded spaces, noises, and social interactions that were beneficial for addressing social anxiety and avoidance. However, other veterans may benefit from cognitive-behavioral treatment that focuses on reframing interpersonal responses and reactions to specific social interactions. Clinical interventions should discern differences between social avoidance resulting from PTSD and social anxiety, a condition that may not be induced by trauma-related experiences. As the results of this case summary are not generalizable and cannot be verified as broadly reaching, this case demonstrates a promising effect of this intervention, particularly for veterans who have comorbid social anxiety and PTSD. Further research would provide more generalizable evidence, with a need for a future randomized control trial to evaluate the intervention with a larger sample of veterans.

\section{Compliance with Ethical Standards}

Conflict of interest The authors declare that they have no conflicts of interest to disclose.

\section{References}

Andrews-Hanna, J. R., Reidler, J. S., Huang, C., \& Buckner, R. L. (2010). Evidence for the default network's role in spontaneous cognition. Journal of Neurophysiology, 104(1), 322-335. https ://doi.org/10.1152/jn.00830.2009.

Barrett, L. F., \& Satpute, A. B. (2013). Large-scale brain networks in affective and social neuroscience: Towards an integrative functional architecture of the brain. Current Opinion in Neurobiology, 23, 361-372. https://doi.org/10.1016/j.conb.2012.12.012.

Blevins, C. A., Weathers, F. W., Davis, M. T., Witte, T. K., \& Domino, J. L. (2015). The Posttraumatic Stress Disorder Checklist for DSM-5 (PCL-5): Development and initial psychometric evaluation. Journal of Traumatic Stress, 28, 489-498. https://doi. org/10.1002/jts.22059.

Boeldt, D., McMahon, E., McFaul, M., \& Greenleaf, W. (2019). Using virtual reality exposure therapy to enhance treatment of anxiety disorders: Identifying areas of clinical adoption and potential obstacles. Frontiers in Psychiatry. https://doi.org/10.3389/fpsyt .2019.00773.

Bressler, S. L., \& Menon, V. (2010). Large-scale brain networks in cognition: Emerging methods and principles. Trends in Cognitive Sciences, 14, 277-290. https://doi.org/10.1016/j.tics.2010.04.004.

Brooke, J. (1996). SUS: A "quick and dirty" usability scale. In P. Jordan, B. Thomas, \& B. Weerdmeester (Eds.), Usability evaluation in industry (pp. 189-194). London: Taylor \& Francis.

Buysse, D. J., Reynolds, C. F., Monk, T. H., Berman, S. R., \& Kupfer, D. J. (1989). The Pittsburgh sleep quality index: A new instrument for psychiatric practice and research. Psychiatry Research, 28(2), 193-213. https://doi.org/10.1016/0165-1781(89)90047-4.

Carl, E., Stein, A. T., Levihn-Coon, A., Pogue, J. R., Rothbaum, B., Emmelkamp, P., et al. (2019). Virtual reality exposure therapy for anxiety and related disorders: A meta-analysis of randomized controlled trials. Journal of Anxiety Disorders, 61, 27-36. https ://doi.org/10.1016/j.janxdis.2018.08.003.

Cavanna, A. E., \& Trimble, M. R. (2006). The precuneus: A review of its functional anatomy and behavioral correlates. Brain, 129(3), 564-583. https://doi.org/10.1093/brain/aw100.

Chen, F., Ke, J., Qi, R., Xu, Q., Zhong, Y., Liu, T., et al. (2018). Increased inhibition of the amygdala by the mPFC may reflect a resilience factor in post-traumatic stress disorder: A resting-state fMRI Granger causality analysis. Frontiers in Psychiatry, 9, 516. https://doi.org/10.3389/fpsyt.2018.00516.

Chesham, R. K., Malouff, J. M., \& Schutte, N. S. (2018). Meta-analysis of the efficacy of virtual reality exposure therapy for social anxiety. Behaviour Change, 35, 152-166. https://doi.org/10.1017/ bec.2018.15.

Chiong, W., Wilson, S. M., D’Esposito, M., Kayser, A. S., Grossman, S. N., Poorzand, P., et al. (2013). The salience network causally influences default mode network activity during moral reasoning. Brain, 136(6), 1929-1941. https://doi.org/10.1093/brain/awt066.

Crowe, S., Cresswell, K., Robertson, A., Huby, G., Avery, A., \& Sheikh, A. (2011). The case study approach. BMC Medical Research Methodology, 11(1), 100-108. https://doi. org/10.1186/1471-2288-11-100.

De Havas, J. A., Parimal, S., Soon, C. S., \& Chee, M. W. (2012). Sleep deprivation reduces default mode network connectivity and anticorrelation during rest and task performance. Neuroimage, 59(2), $1745-1751$.

Delgado, M. R., Nearing, K. I., LeDoux, J. E., \& Phelps, E. A. (2008). Neural circuitry underlying the regulation of conditioned fear and its relation to extinction. Neuron, 59(5), 829-838. https:// doi.org/10.1016/j.neuron.2008.06.029.

Dolan, J. E. (2016). Splicing the divide: A review of research on the evolving digital divide among K-12 students. Journal of 
Research on Technology in Education, 48(1), 16-37. https://doi. org/10.1080/15391523.2015.1103147.

Ebert, D. D., Van Daele, T., Nordgreen, T., Karekla, M., Compare, A., Zarbo, C., et al. (2018). Internet- and mobile-based psychological interventions: Applications, efficacy, and potential for improving mental health: A report of the EFPA E-Health Taskforce. European Psychologist, 23(2), 167-187. https://doi.org/10.1027/10169040/a000318.

Etkin, A., \& Wager, T. D. (2007). Functional neuroimaging of anxiety: A meta-analysis of emotional processing in PTSD, social anxiety disorder, and specific phobia. American Journal of Psychiatry, 164, 1476-1488. https://doi.org/10.1176/appi.ajp.2007.07030504.

First, M. B., Williams, J. B. W., Karg, R. S., \& Spitzer, R. L. (2015). Structured Clinical Interview for DSM-5: Research Version. Philadelphia: American Psychiatric Association.

Foa, E. B., McLean, C. P., Zang, Y., Rosenfield, D., Yadin, E., Yarvis, J. S., et al. (2018). Effect of prolonged exposure therapy delivered over 2 weeks vs 8 weeks vs present-centered therapy on PTSD symptom severity in military personnel: A randomized clinical trial. Journal of the American Medical Association, 319(4), 354364. https://doi.org/10.1001/jama.2017.21242.

Foa, E. B., \& Kozak, M. J. (1986). Emotional processing of fear: Exposure to corrective information. Psychological Bulletin, 99(1), 20-35. https://doi.org/10.1037/0033-2909.99.1.20.

Frank, L. H., Kennedy, R. S., Kellogg, M. E., McCauley, M. E. (1983). Simulator sickness: a reaction to a transformed perceptual world. 1 Scope of the Problem (NAVTRAEQUIPCEN TN-65) Naval Training Equipment Center, Orlando, FL.

Freeman, D., Reeve, S., Robinson, A., Ehlers, A., Clark, D., Spanlang, B., et al., (2017). Virtual reality in the assessment, understanding, and treatment of mental health disorders. Psychological Medicine, 47(14), 2393-2400. https://doi.org/10.1017/S003329171 $700040 X$.

Garrett, A., Cohen, J. A., Zack, S., Carrion, V., Jo, B., Blader, J., et al. (2019). Longitudinal changes in brain function associated with symptom improvement in youth with PTSD. Journal of Psychiatric Research, 114, 161-169. https://doi.org/10.1016/j.jpsychires .2019.04.021.

Gavgani, A. M., Walker, F. R., Hodgson, D. M., \& Nalivalko, E. (2018). A comparative study of cybersickness during exposure to virtual reality and "classic" motion sickness: Are they different? Journal of Applied Physiology, 125, 1670-1680. https://doi. org/10.1152/japplphysiol.00338.2018.

Gonçalves, R., Pedrozo, A.L., Coutinho, E.S.F., Figueira, I., \& Ventura, P. (2012). The efficacy of virtual reality exposure therapy in treatment of PTSD: A systematic review. PLoS ONE, 7(12), 1-7. https://doi.org/10.1371/journal.pone.0048469.

Harvey, A. G., Jones, C., \& Schmidt, D. A. (2003). Sleep and posttraumatic stress disorder: A review. Clinical Psychology Review, 23(3), 377-407. https://doi.org/10.1016/s0272-7358(03)00032-1.

Jerdan, S. W., Grindle, M., Van Woerden, H. C., \& Kamel Boulos, M. N. (2018). Head-mounted virtual reality and mental health: Critical review of current research. Journal of Medical Internet Research. https://doi.org/10.2196/games.9226.

Johnstone, T., Van Reekum, C. M., Urry, H. L., Kalin, N. H., \& Davidson, R. J. (2007). Failure to regulate: Counterproductive recruitment of top-down prefrontal-subcortical circuitry in major depression. Journal of Neuroscience, 27(33), 8877-8884. https://doi. org/10.1523/JNEUROSCI.2063-07.2007.

Jones, D. J., Anton, M., Gonzalez, M., Honeycutt, A., Khavjou, O., Forehand, R., \& Parent, J. (2015). Incorporating mobile phone technologies to expand evidence-based care. Cognitive and Behavioral Practice, 22(3), 281-290. https://doi.org/10.1016/j. cbpra.2014.06.002j.

Kim, H. E., Hong, Y.-J., Kim, M.-K., Jung, Y. H., Kyeong, S., \& Kim, J.-J. (2017). Effectiveness of self-training using the mobile-based virtual reality program in patients with social anxiety disorder. Computers in Human Behavior, 73, 614-619. https://doi. org/10.1016/j.chb.2017.04.017.

King, A., Angstadt, M., Sripada, C., \& Liberzon, I. (2017). Increased Default Mode Network (DMN) connectivity with attention networks with a mindfulness-based intervention for PTSD: Seed and whole brain connectomics analyses. Biological Psychiatry, 81(10), S43-S44. https://doi.org/10.1016/j.biopsych.2017.02.115.

King, A. P., Block, S. R., Sripada, R. K., Rauch, S., Giardino, N., Favorite, T., et al. (2016). Altered default mode network (DMN) resting state functional connectivity following a mindfulnessbased exposure therapy for posttraumatic stress disorder (PTSD) in combat veterans of Afghanistan and Iraq. Depression and Anxiety, 33(4), 289-299. https://doi.org/10.1002/da.22481.

Klumpp, H., Hosseini, B., \& Phan, K. L. (2018). Self-reported sleep quality modulates amygdala resting-state functional connectivity in anxiety and depression. Frontiers in Psychiatry. https://doi. org/10.3389/fpsyt.2018.00220.

Kothgassner, O. D., Goreis, A., Kafka, J. X., Van Eickels, R. L., Plener, P. L., \& Felnhofer, A. (2019). Virtual reality exposure therapy for posttraumatic stress disorder (PTSD): A meta-analysis. European Journal of Psychotraumatology. https://doi.org/10.1080/20008 198.2019.1654782

Linardon, J., Cuijpers, P., Carlbring, P., Messer, M., \& Fuller, F.-T. (2019). The efficacy of app-supported smartphone interventions for mental health problems: A meta-analysis of randomized controlled trials. World Psychiatry, 8(3), 325-336. https://doi. org/10.1002/wps.20673.

Mclay, R. N., Klam, W. P., \& Volkert, S. L. (2010). Insomnia is the most commonly reported symptom and predicts other symptoms of post-traumatic stress disorder in U.S. service members returning from military deployments. Military Medicine, 175(10), 759762. https://doi.org/10.7205/milmed-d-10-00193.

McLean, C. P., \& Foa, E. B. (2011). Prolonged exposure therapy for post-traumatic stress disorder: A review of evidence and dissemination. Expert Review of Neurotherapeutics, 11(8), 1151-1163. https://doi.org/10.1586/ern.11.94.

McMillan, K. A., Asmundson, G. J. G., \& Jitender, S. (2017). Comorbid PTSD and social anxiety disorder: Associations with quality of life and suicide attempts. The Journal of Nervous and Mental Disease, 205(9), 732-737. https://doi.org/10.1097/NMD.00000 00000000704.

Menon, V. (2011). Large-scale brain networks and psychopathology: A unifying triple network model. Trends in Cognitive Sciences, 15(10), 483-506. https://doi.org/10.1016/j.tics.2011.08.003.

Metsis, V., Lawrence, G., Trahan, M. H., Smith, K. S., Tamir, D., \& Selber, K. (2019). $360^{\circ}$ video: A prototyping process for developing virtual reality interventions. Journal of Technology in Human Services, 37(1), 32-50. https://doi.org/10.1080/15228 835.2019.160429.

Morley, R. H., Jantz, P. B., \& Graham, R. (2019). The role of the Salience Network in perceptions of hostility and aggression. Neuropsychological Trends. https://doi.org/10.7358/ neur-2019-025-morl.

Nason, E., Trahan, M. H., Smith, K. S., Metsis, V., \& Selber, K. (2019). Virtual treatment for veteran social anxiety disorder: A comparison of $360^{\circ}$ video and $3 \mathrm{D}$ virtual reality using two case studies. Journal of Technology in Human Services, 38(3), 288-303. https ://doi.org/10.1080/15228835.2019.1692760.

Opris, D., Pintea, S., Garcia-Palacios, A. G., Botella, C., Szamoskozi, S., \& David, D. (2012). Virtual reality exposure therapy in anxiety disorders: A quantitative analysis. Depression and Anxiety, 29, 85-93.

Powers, M. B., \& Emmelkamp, P. M. G. (2008). Virtual reality exposure therapy for anxiety disorders: A meta-analysis. Journal of Anxiety Disorders, 22, 561-569. 
Prinz, U., Nutzinger, D. O., Schulz, H., Petermann, F., Braukhaus, C., \& Andreas, S. (2013). Comparative psychometric analyses of the SCL-90-R and its short versions in patients with affective disorders. BMC Psychiatry. https://doi. org/10.1186/1471-244X-13-104.

Rauch, S. L., Milad, M. R., Orr, S. P., Quinn, B. T., Fischl, B., \& Pitman, R. K. (2005). Orbitofrontal thickness, retention of fear extinction, and extraversion. NeuroReport, 16(17), 1909-1912. https://doi.org/10.1097/01.wnr.0000186599.66243.50.

Richards, A., Kanady, J. C., \& Neylan, T. C. (2019). Sleep disturbance in PTSD and other anxiety-related disorders: An updated review of clinical features, physiological characteristics, and psychological and neurobiological mechanisms. Neuropsychopharmacology, 45(1), 55-73. https://doi.org/10.1038/s41386-019-0486-5.

Rodrigues, N. A., Smith, K. S., Selber, K., \& Russian, C. J. (2019). Identifying sleep as a health need in student veterans. Pulmonology and Respiratory Medicine, 8(7), 531-546.

Rothbaum, B.O., Hodges, L., Alarcon, R., Ready, D., Shahar, F., Graap, K., et al. (1999). Virtual reality exposure therapy for PTSD Vietnam veterans: A case study. Journal of Traumatic Stress, 12(2), 263-271.

Rothbaum, B. O., Hodges, L. F., Ready, D., Graap, K., \& Alacron, R. D. (2001). Virtual reality exposure therapy for Vietnam veterans with posttraumatic stress disorder. The Journal of Clinical Psychiatry, 62(8), 617-622.

Schmidt, N. B., \& Keough, M. E. (2010). Treatment of panic. Annual Review of Clinical Psychology, 6, 241-256. https://doi. org/10.1146/annurev.clinpsy.121208.131317.

Seeley, W. W., Menon, V., Schatzberg, A. F., Keller, J., Kenna, H., Reiss, A. L., et al. (2007). Dissociable intrinsic connectivity networks for salience processing and executive control. The Journal of Neuroscience, 27(9), 2349-2356. https://doi.org/10.1523/ JNEUROSCI.5587-06.2007.

Sripada, R. K., King, A. P., Welsh, R. C., Garfinkel, S. N., Wang, X., Sripada, C. S., \& Liberzon, I. (2012). Neural dysregulation in posttraumatic stress disorder: Evidence for disrupted equilibrium between salience and default mode brain networks. Psychosomatic Medicine, 74(9), 904. https://doi.org/10.1097/PSY.0b013e3182 $73 b f 33$.

Tellegen, A., \& Atkinson, G. (1974). Openness to absorbing and selfaltering experiences ("absorption"), a trait related to hypnotic susceptibility. Journal of Abnormal Psychology, 83, 268-277.

Trahan, M. H., Ausbrooks, A., Smith, K. S., Metsis, V., Berek, A., Trahan, L., \& Selber, K. (2019). Experiences of student veterans with social anxiety. Social Work in Mental Health, 17(2), 197-221. https://doi.org/10.1080/15332985.2018.1522607.

Urry, H. L., van Reekum, C. M., Johnstone, T., Kalin, N. H., Thurow, M. E., Schaefer, H. S., et al. (2006). Amygdala and ventromedial prefrontal cortex are inversely coupled during regulation of negative affect and predict the diurnal pattern of cortisol secretion among older adults. Journal of Neuroscience, 26(16), 4415-4425.

Van den Heuvel, M. P., \& Sporns, O. (2011). Rich-club organization of the human connectome. The Journal of
Neuroscience, 31, 15775-15786. https://doi.org/10.1523/JNEUR OSCI.3539-11.2011.

Van den Heuvel, M. P., \& Sporns, O. (2013). Network hubs in the human brain. Trends in Neurocognitive Sciences, 17, 683-696. https://doi.org/10.1016/j.tics.2013.09.012.

Van Stegeren, A. H., Wolf, O. T., Everaerd, W., Scheltens, P., Barkhof, F., \& Rombouts, S. A. (2007). Endogenous cortisol level interacts with noradrenergic activation in the human amygdala. Neurobiology of Learning and Memory, 87(1), 57-66. https://doi. org/10.1016/j.nlm.2006.05.008.

Wall, C. L., \& Lowe, M. (2020). Facing the fear: Resilience and social support in veterans and civilians with PTSD. Journal of Aggression, Conflict and Peace Research, 12(2), 75-85.

Watson, N. F., Badr, M. S., Belenky, G., Bliwise, D. L., Buxton, O. M., Buysse, D., et al. (2015). Recommended amount of sleep for a healthy adult: A joint consensus statement of the American Academy of Sleep Medicine and Sleep Research Society. Sleep, 38(6), 843-844. https://doi.org/10.5665/sleep.4716.

Weathers, F. W., Litz, B. T., Keane, T. M., Palmieri, P. A., Marx, B. P., \& Schnurr, P. P. (2013). The PTSD Checklist for DSM-5 (PCL5). Scale available from the National Center for PTSD at www. ptsd.va.gov.

Wechsler, T. F., Mühlberger, A., \& Kümpers, F. (2019). Inferiority or even superiority of virtual reality exposure therapy in phobias? A systematic review and quantitative meta-analysis on randomized controlled trials specifically comparing the efficacy of virtual reality exposure to gold standard in vivo exposure in agoraphobia, specific phobia and social phobia. Frontiers in Psychology, 10, 1758. https://doi.org/10.3389/fpsyg.2019.01758.

Witmer, B.G., \& Singer, M.J. (1998). Measuring presence in virtual environments: A presence questionnaire. Presence: Teleoperators \& Virtual Environments, 7(3), 225-240.

Wolf, R. C., \& Herringa, R. J. (2016). Prefrontal-amygdala dysregulation to threat in pediatric posttraumatic stress disorder. Neuropsychopharmacology, 41(3), 822-831. https://doi.org/10.1038/ npp.2015.209.

Yin, R. K. (1984). Case study research and applications: Design and methods (6th ed.). Sage.

Yoo, S., Hu, P. T., Gujar, N., Jolesz, F. A., \& Walker, M. P. (2007). A deficit in the ability to form new human memories without sleep. Nature Neuroscience, 10(3), 385-392. https://doi.org/10.1038/ $\mathrm{nn} 1851$.

Publisher's Note Springer Nature remains neutral with regard to jurisdictional claims in published maps and institutional affiliations.

Mark H. Trahan The Virtual Reality Technology Lab at Texas State University (https://www.socialwork.txstate.edu/centers-institutes/ VRTL.html) is an interdisciplinary laboratory of faculty from social work, psychology, communication design, and computer science. 